\title{
$\Omega$
}

Evangelia Panagiotidou' ${ }^{1}$ Serafeim-Chrysovalantis Kotoulas' ${ }^{1}$,

Maria Kilmpasani², Nikoleta Pastelli², Sofia Akritidou',

Evangelos Chatzopoulos' ${ }^{1}$ Vasilis Bikos ${ }^{1}$, Vasilios Bagalas',

Katalin Fekete-Passa ${ }^{1}$, loannis Stanopoulos ${ }^{1,3}$, Athanasia Pataka ${ }^{1,3}$

(1]

${ }^{1}$ Respiratory Failure Unit, General Hospital of Thessaloniki “G. Papanikolaou”, Thessaloniki, Greece.

'Laboratory of Pathology, General Hospital of Thessaloniki "G. Papanikolaou”, Thessaloniki, Greece.

3Medical School, Aristotle University of Thessaloniki, Thessaloniki, Greece.

\section{A 78-year-old female who presents with a non-resolving pneumonia: what is your diagnosis?}

\section{Case report}

A 78-year-old white female (body mass index $32.3 \mathrm{~kg} \cdot \mathrm{m}^{-2}$ ) presented with low grade fever, cough, dyspnoea and weakness that did not resolve 2 weeks after being treated with moxifloxacin for a lower pulmonary tract infection (7-day regimen). Her medical history included bronchial asthma, hypothyroidism and gastro-oesophageal reflux. She lived in her house, had never smoked and had no prior hospitalisations.

On clinical examination, she presented with fever $\left(38.2^{\circ} \mathrm{C}\right)$, normal blood pressure $(125 / 72 \mathrm{mmHg})$, tachycardia (105 beats $\cdot \mathrm{min}^{-1}$ ), elevated respiratory rate $\left(26\right.$ breaths $\cdot \mathrm{min}^{-1}$ ) and low oxygen saturation on room air (88\%). On chest auscultation, crackles and diminished lung sounds were present in the base of the right lung. She did not present jugular venous enlargement or leg oedemas and the cardiac examination was normal. Examination of the lymph nodes and abdomen was also normal.

\section{Laboratory examinations}

Mild leukocytosis (12450 cells $\left.\cdot \mathrm{dL}^{-1}\right)$, with neutrophilic predominance (92\%) and elevated C-reactive protein (CRP) $\left(17 \mathrm{mg} \cdot \mathrm{L}^{-1}\right)$ were suggestive of an infectious condition. Blood gas analysis showed mild hypoxaemia on room air $(\mathrm{pH} 7.41$, oxygen tension $58 \mathrm{mmHg}$, carbon dioxide tension $32 \mathrm{mmHg}$ ) and the patient received oxygen through nasal cannula (inspiratory oxygen fraction 35\%) to correct her oxygenation. Spirometry revealed severe restriction (forced vital capacity (FVC) 1.15 L (33.5\% pred), forced expiratory volume in $1 \mathrm{~s}\left(\mathrm{FEV}_{1}\right) 0.76 \mathrm{~L}$ (29.3\% pred), FEV1/FVC 88.3\%).

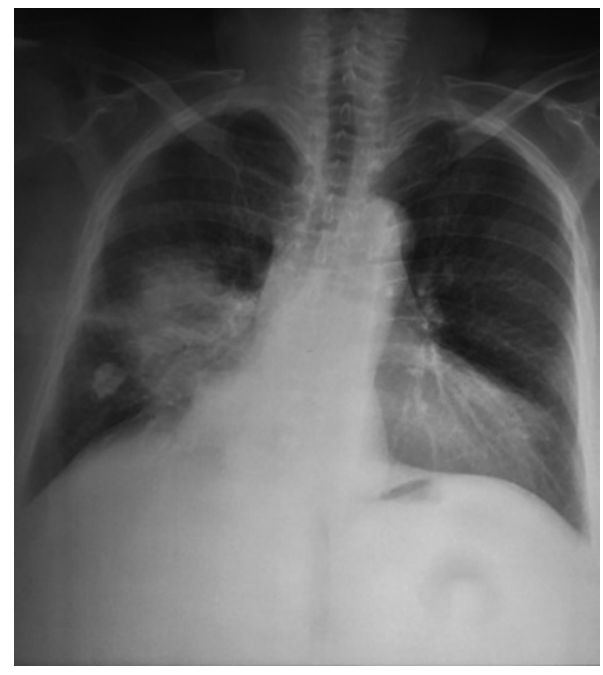

Figure 1 Chest radiograph on admission.
Cite as: Panagiotidou $\mathrm{E}$, Kotoulas SC, Kilmpasani M, et al. A 78-year-old female who presents with a nonresolving pneumonia: what is your diagnosis? Breathe 2018; 14: e123-e127.

@ERSpublications

Non-resolving pneumonia with a mass-like radiological appearance raises clinical suspicion of many causes, with malignancy the most prominent. Infectious causes should be investigated thoroughly as they carry a better prognosis. http://ow.ly/QEPL30mLIk4 

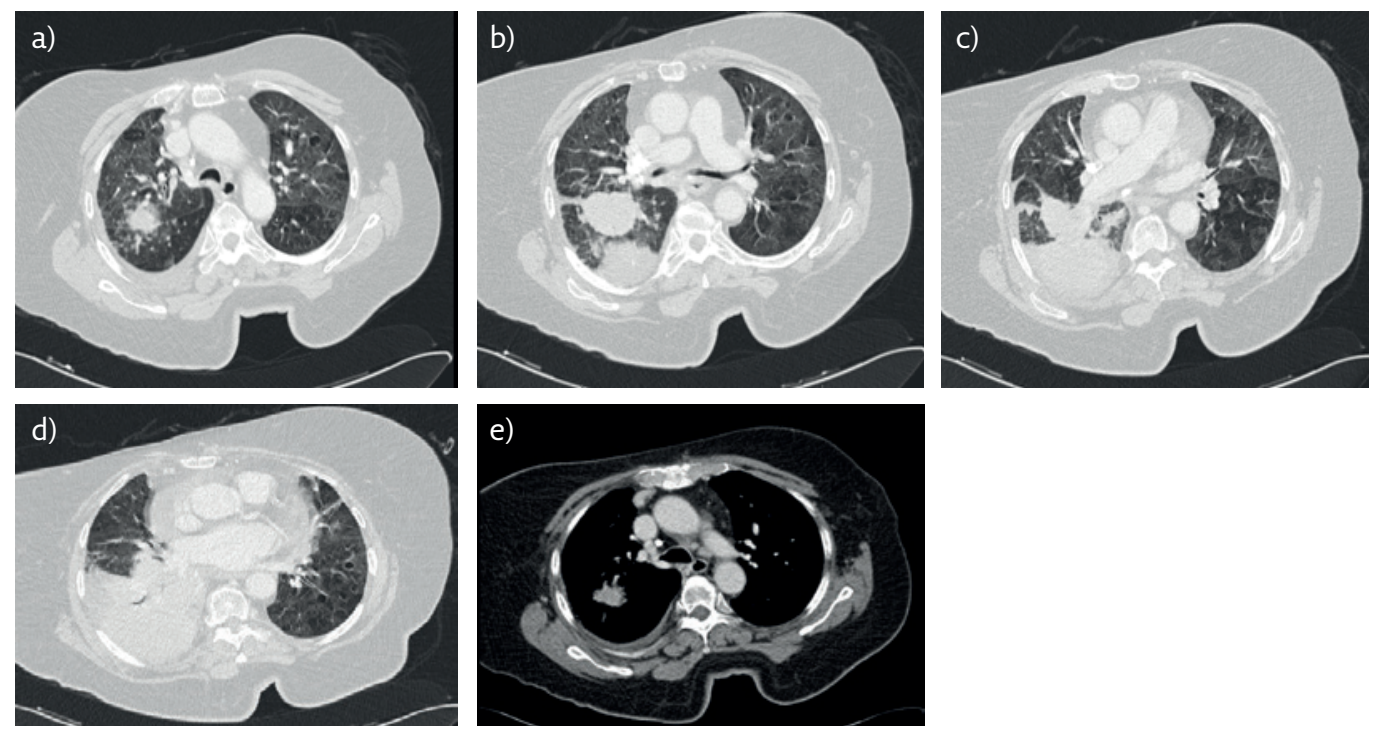

Figure 2 Chest CT on admission. $a-d$ ) lung window, e) mediastinal window.

Sputum cultures for common pathogens, and tuberculous and nontuberculous mycobacteria were negative.

\section{Investigations}

A chest radiograph (figure 1 ) and then a chest computed tomography (CT) were ordered (figure 2).

Task 1

Can you describe the findings in the chest radiograph?

Task 2

Can you describe the findings in the chest $\mathrm{CT}$ ?

Task 3

What is the most possible differential diagnosis? 


\section{Answer 1}

The chest radiograph revealed segmental consolidation with a silhouette sign of the right cardiac border with possible cavitation and a nodule in the right lower lung zone (figure 1). The major possible diagnoses are cancer and pneumonia.

\section{Answer 2}

A mass of $8.7 \mathrm{~cm}$ with an abnormal circumference was present in the posterior segment of the right upper lobe and extended to the right lower lobe, causing obstruction of the bronchus for the right lower lobe and atelectasis (figure 2). In addition, a calcified granuloma of $1.8 \mathrm{~cm}$ in the right middle lobe (figure 2a) and slightly enlarged mediastinal lymph nodes were found (figure $2 \mathrm{e}$ ).

\section{Answer 3}

Possible diagnoses for a pulmonary mass are listed in table 1.

The chest radiograph revealed a focal opacity with possible cavitation and a silhouette sign of the right cardiac border (figure 1). Pneumonia and a lung tumour were considered as the most probable diagnoses. Other causes of pulmonary mass-like opacity are presented in table 1 [1-3].

Chest CT revealed a mass of $8.7 \mathrm{~cm}$ with an abnormal circumference in the posterior segment of the right upper lobe that extended to the right lower lobe, causing obstruction of the bronchus for the right lower lobe and atelectasis. A calcified granuloma of $1.8 \mathrm{~cm}$ in the right middle lobe and slightly enlarged mediastinal lymph nodes (figure 2 ) were also found.

Table 1 Differential diagnosis of a pulmonary mass

Bacterial infection

Fungal infection

Tuberculosis

Infection from nontuberculous mycobacteria

Lung abscess

Primary lung tumour

Secondary lung tumour

Inflammatory pseudo-tumour

Cryptogenic organising pneumonia

Round atelectasis

Hydatid cyst

Haematoma

Pulmonary arteriovenous malformation
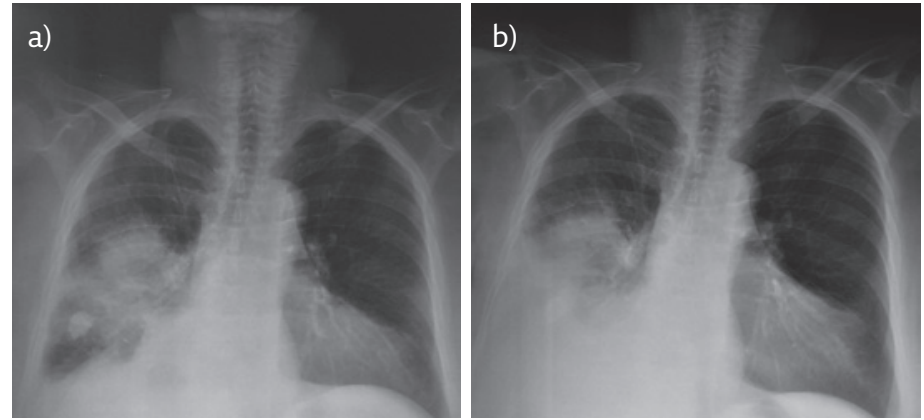

Figure 3 a) Chest radiograph after 10 days of antibiotic treatment showing enlargement of the consolidation and pleural effusion. b) Chest radiograph after 12 days of antibiotic treatment showing further deterioration.

\section{What would be the next step in the management of this patient?}

Fibreoptic bronchoscopy would be the next appropriate step in order to collect specimens for culture for common pathogens or tuberculous mycobacteria as a diagnosis of a poorly treated lower tract infection was possible (post-obstructive pneumonia). In addition, bronchoalveolar lavage, cytology and biopsy could help in the diagnosis of a possible malignancy. As all the results from the bronchoscopic specimens were negative, a chest CT-guided fine needle biopsy was performed. The results were negative for neoplastic or granulomatosis findings, but chronic inflammation and fibrosis were reported.

The patient was hospitalised to receive intravenous piperacillin-tazobactam, but the low-grade fever $\left(38^{\circ} \mathrm{C}\right)$ did not resolve. Lung
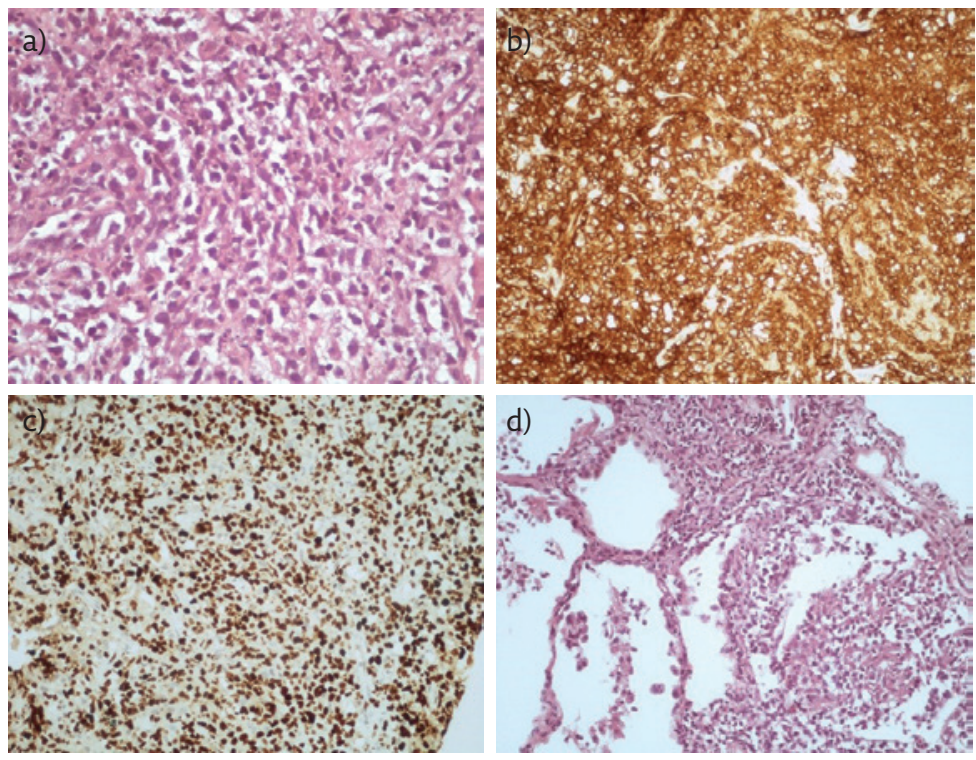

Figure 4 a) The neoplastic cells have medium-to-large size, abundant cytoplasm and enlarged, irregular nuclei. b) Immunohistochemical stain for CD79a is positive, demonstrating the B-cell origin of the neoplastic cells. c) Proliferation marker Ki67 is positive in 80-90\% of the neoplastic cells. d) Diffuse proliferation of large neoplastic lymphoid cells, infiltrating the lung parenchyma. Magnification: a) $\times 40 ; b-d) \times 20$. 
auscultation deteriorated in the right lung; white blood cells increased ( 18900 cells $\cdot \mathrm{dL}^{-1}$ ) along with CRP $\left(21 \mathrm{mg} \cdot \mathrm{L}^{-1}\right)$ and procalcitonin $\left(2.1 \mathrm{ng} \cdot \mathrm{mL}^{-1}\right)$. The chest radiography was repeated and revealed enlargement of the consolidation and pleural effusion, after 10 days of intravenous antibiotic treatment (figure 3).

Thoracentesis was performed and revealed an exudative pleural effusion $(\mathrm{pH} 7.37$, glucose $\left.91 \mathrm{mg} \cdot \mathrm{dL}^{-1}\right)$. Cultures and cytology of the pleural fluid were negative.

As the patients' condition deteriorated, a second chest CT-guided biopsy was performed that revealed a diffuse large $B$-cell lymphoma, with a high degree of malignancy (figure 4)

\section{Discussion}

Our patient presented with a non-resolving pneumonia with a mass-like radiological appearance. A thorough investigation of the possible causes was warranted. The first radiograph of the patient in adjunct to the acute onset of fever and malaise was interpreted as a lower tract infection. CT was performed after failure to treat with a double antibiotic regimen and guided the clinical suspicion to a pulmonary mass (table 1) that was accompanied by atelectasis, as well as by mild mediastinal lymphadenopathy. The most possible differential diagnoses were: 1) pneumonia of infectious origin, 2) cryptogenic organising pneumonia, and 3) lung tumour complicated with organising pneumonia [1-3].

Organising pneumonia [1] was considered a possible diagnosis as it has a subacute onset and mimics an infectious disorder in both clinical presentation and radiological appearance. It may present either as idiopathic organising pneumonia, termed "cryptogenic", or as secondary organising pneumonia in multiple disorders such as infection, connective tissue disease, haematological malignancy and cancer, and may occur distal to airway obstruction [1]. A thoracoscopic lung biopsy, characterised by intra-alveolar granulation tissue with fibroblasts, myofibroblasts and loose connective matrix, is recommended for a definite diagnosis, although it is not always conducted in clinical practice. Alternatively, fibreoptic bronchoscopy with bronchoalveolar lavage and transbronchial biopsy can offer important information and usually presumptive treatment with corticosteroids is initiated. In our case, the histopathological findings were not suggestive of organising pneumonia.

Transthoracic lung biopsy revealed primary lymphoma of the lung (PPL), which is a rare condition with a prevalence of $0.3 \%$ of all primary pulmonary neoplasms [4, 5]. Diffuse large B-cell lymphoma represents the second most common type of PPL with a prevalence of $12-20 \%$ and a median survival of 8-10 years. It can occur in both immunocompetent and immunocompromised patients, such as solid organ transplant recipients and HIV patients. Patients manifest fever, weight loss, cough, dyspnoea and rarely haemoptysis. Radiological examinations usually show a single pulmonary mass or atelectasis. Pleural effusion is also often present. Bronchial, transbronchial biopsy or CT-guided transthoracic needle biopsy can yield promising results. Histology reveals blast-like lymphoid cells with strong mitotic activity and necrosis (figure 4d). In adjunct, immunohistochemistry is performed to rule out primary or metastatic carcinoma, melanoma or other epithelial neoplasms in cases with extensive fibroblastic reaction, nodal or leukaemic involvement. Treatment is based on surgical resection and adjunctive combination chemotherapy regimens $[4,5]$. Our patient received chemotherapy, as she was not a candidate for surgery due to poor performance status, severe restrictive lung function and involvement of mediastinal lymph nodes. She died 8 months after the diagnosis.

Key points

- Non-resolving pneumonia with a mass-like radiological appearance raises clinical suspicion of many causes, with malignancy the most prominent.

- Infectious causes should be investigated thoroughly as they carry a better prognosis.

- PPL is a rare condition, but it should be included in the differential diagnosis of a lung mass. Biopsy is needed for the diagnosis and the choice of treatment.

\section{Conflict of interest}

None declared.

\section{References}

1. Spiro S, Stephen G, Silvestri GA, et al. Clinical Respiratory Medicine E-Book: Expert Consult-Online and Print. Elsevie Health Sciences, 2012.
2. Agrons GA, Rosado-de-Christenson ML, Kirejczyk WM, et al. Pulmonary inflammatory pseudotumor: radiologic features. Radiology 1998; 206: 511-518. 
3. Gould MK, Fletcher J, lannettoni MD, et al. Evaluation of patients with pulmonary nodules: when is it lung cancer? ACCP evidence-based clinical practice guidelines (2nd edition). Chest 2007; 132: Suppl., 108S-130S.
4. Cadranel J, Wislez M, Antoine M. Primary pulmonary lymphoma. Eur Respir J 2002; 20: 750-762.

5. William J, Variakojis D, Yeldandi A, et al. Lymphoproliferative neoplasms of the lung: a review. Arch Pathol Lab Med 2013; 137: 382-391. 